\section{Cystic fibrosis in a 70 year old woman}

\author{
P van Biezen, S E Overbeek, C Hilvering
}

\begin{abstract}
A 68 year old woman with a lifelong history of chronic bronchitis was diagnosed as having cystic fibrosis. The diagnosis was based on a suggestive family history, steatorrhoea, bronchiectasis with respiratory insufficiency, and very high sweat sodium content. The patient was found to be heterozygous for the delta F 508 gene defect.
\end{abstract}

Cystic fibrosis is regarded as a disease of childhood and more than $95 \%$ of patients have the diagnosis made in childhood. We present a woman with a long history of respiratory infections who was diagnosed as having cystic fibrosis at the age of 68 years. This case illustrates that cystic fibrosis should be considered in all age groups.

\section{Case report}

The patient, a Dutch woman, was born in 1920. From early childhood she had suffered from a cough, producing thick, purulent sputum every day and having episodes of haemoptysis. Her symptoms diminished in her 20 s but she still occasionally had attacks of

Accepted 10 September 1991 bronchitis. At the age of 36 she underwent a cholecystectomy. After the age of 50 her pulmonary symptoms and function worsened and she frequently required medical treatment. During the last two decades Staphylococcus aureus and Haemophilus influenzae were isolated repeatedly from her sputum. Pancreatic insufficiency was shown by a high fat loss in her stools, but she had no symptoms of malabsorption and needed no pancreatic enzyme substitution. Over the years her pulmonary function declined, finally resulting in the use of corticosteroid and supplementary oxygen treatment at home. In 1988, at the age of 68 years, she was admitted to our department because of an exacerbation of dyspnoea and a productive cough. Three of her sisters had died, aged from 6 weeks to 6 months, because of "sticky mucus." She is married, had uncomplicated pregnancies, and has two healthy sons. Her children and grandchildren have had no respiratory or gastrointestinal symptoms. Physical examination showed a 68 year old well nourished woman (height $163 \mathrm{~cm}$, weight $65 \mathrm{~kg}$ ). Her pulse rate and blood pressure were normal. The nasal passages were clear and there were no signs of chronic sinusitis, clubbing, or ankle oedema. Coarse crackles were heard over both lungs and especially in the upper parts. Heart sounds were normal and abdominal examination showed no abnormality.

Glucose and haemoglobin concentrations and results of liver and renal function tests were normal. The fat soluble vitamin concentrations were low. Arterial blood gas analysis showed that the $\mathrm{pH}$ was $7 \cdot 36$, oxygen tension $\left(\mathrm{PaO}_{2}\right) 8.4 \mathrm{kPa}$, carbon dioxide tension $\left(\mathrm{PaCO}_{2}\right)$ $7.8 \mathrm{kPa}$, and oxygen saturation $\left(\mathrm{SaO}_{2}\right) 91 \%$ while she was having $1.5 \mathrm{l} / \mathrm{min}$ oxygen. Chest radiograph (figure) showed peribronchial thickening, bronchiectasis, and reduction of the volume of the upper lobes. A sinus radiograph showed no evidence of sinusitis.

Pulmonary function tests showed a combined restrictive and obstructive defect (vital capacity 0.991 ( $37 \%$ of predicted) FEV 0.401 ( $19 \%$ of predicted, with no reversibility after bronchodilator).

Haemophilus influenzae and later Pseudomonas aeruginosa were isolated from sputum. Faecal fat excretion was about $53 \mathrm{~g}$ over 72 hours with a fat intake of $240 \mathrm{~g}$ (normal loss with a $80 \mathrm{~g} /$ day intake should be under $8 \mathrm{~g}$ / day. The sweat sodium concentration (the patient had been taking $10 \mathrm{mg}$ oral prednisone daily for many years) was $94 \mathrm{mmol} / \mathrm{l}$ for both the right and the left arm in samples of respectively 106 and $139 \mathrm{mg}$ of sweat.

This combination of findings and a suggestive family history suggested the diagnosis of cystic fibrosis.

The patient proved to be a compound heterozygote with the genotype delta F508/ unknown. Other probes tested were G542 x, d I 507, G 551 d, S 594 N and S 549 I. Other family members refused genetic analysis as they had no complaints.

She was treated with antibiotics, oxygen, and chest physiotherapy. Over the past three 
years the patient has had frequent admissions to hospital with pulmonary problems but is in a stable condition.

\section{Discussion}

Cystic fibrosis is an autosomal recessive genetic disorder. Recently the cystic fibrosis locus has been located on chrosome 7 and the nucleotide base sequence of the gene has been determined. The most frequently reported mutation is delta F508. ${ }^{1}$ Cystic fibrosis includes chronic obstructive and suppurative lung disease, exocrine pancreatic deficiency and abnormalities of the liver and of the reproductive tract. ${ }^{23}$ The diagnosis is based on the detection of increased concentrations of electrolytes in the sweat. ${ }^{4}$ There is often a family history of cystic fibrosis. Expression of the disease varies and there is a little relation between age at diagnosis and age at death. A few elderly patients with cystic fibrosis have been reported, ${ }^{5-8}$ but our patient is probably the oldest to be diagnosed and reported. This case shows that in patients with appropriate symptoms cystic fibrosis should be considered whatever their age. A mild clinical course may delay diagnosis and may be related to heterozygosity.

We thank Dr D J J Halley and Dr H J Veeze of the department of clinical genetics of the Universital Hospital Rotterdam for their analysis and study of the genetic disorder.

1 Kerem B, Rommens JH, Buchanan JA, et al . Identification of the cystic fibrosis gene: genetic analysis. Science 1989;245:1073-80.

2 Neyens HJ, Sinaasappel $M$, de Groot $R$, de Jongste JC Overbeek SE. Cystic fibrosis, pathophysiological and clinical aspects. Eur J Pediatr 1990;149:742-51.

3 Penketh ARL, Wise A, Mearns HB, Hodson ME, Batton JC Cystic fibrosis in adolescents and adults. Thorax 1987; 42:526-32.

4 Gibson LE, Cooke RE. A test for concentrations of electrolytes in sweat in cystic fibrosis of the pancreas utilizing pilocarpine by iontophoresis. Pediatrics 1959;23:545-9.

5 Brown RF, Di Benedett R, Russell D, Su C. Variant cystic fibrosis in an elderly man. South Med J 1986;79:1430-2.

6 Evensen SA. A 69-year old man with chronic obstructive pulmonary disease, pancreatic insufficiency and elevated sweat electrolytes. Acta Med Scand 1981;209:141-3.

7 Hunt T, Geddes B. Newly diagnosed cystic fibrosis in middle and later life. Thorax 1985;40:23-6.

8 Su CT, Beanblossom B. Typical cystic fibrosis in an elderly woman. Am J Med 1989;86:701-3.

\section{Removal of endobronchial mucormycosis lesion through a rigid bronchoscope}

\author{
Sulaiman Al-Majed, Feisal Al-Kassimi, \\ Mohamad Ashour, Mohamad Osman \\ Mekki, Saleem Sadiq
}

\begin{abstract}
A 44 year old man with poorly controlled diabetes mellitus developed endobronchial mucormycosis, which totally obstructed the right lower lobe bronchus. The lesion was removed through a rigid bronchoscope. Two weeks later the bronchus was free of mucormycosis histologically and on culture.
\end{abstract}

Pulmonary mucormycosis is a rare fungal infection that occurs primarily in patients with poorly controlled diabetes. ${ }^{12}$ Acidosis appears to enhance fungal growth. ${ }^{3}$ Rhizomucor, Rhizopus, and Absidia are the commonly encountered genera. All the organisms have a predilection for invading blood vessels and causing thrombosis and infarction, ${ }^{4}$ thus explaining the high incidence of haemoptysis.

Endobronchial mucormycosis is well des- cribed and is known to cause severe or fatal haemoptysis and asphyxiation. ${ }^{5}$ Early aggressive surgical resection of airway mucormycosis is therefore recommended. ${ }^{56}$

We report a patient in whom endobronchial mucormycosis was removed endoscopically.

\section{Case report}

A 44 year old man was admitted to King Khalid University Hospital, Riyadh, with fever, sweating, cough with grey sputum, and nonpleuritic chest pain of a week's duration. He had had diabetes for 10 years. His diabetes had been poorly controlled since he stopped his regular insulin injections three months before admission. On examination his temperature was $38.8^{\circ} \mathrm{C}$ and respiratory rate $22 / \mathrm{min}$. There was dullness to percussion in the right lower zone, with bronchial breathing and crackles. The chest radiograph showed shadowing with loss of volume in the right lower lobe. Pneumonia was diagnosed. Plasma glucose was $32.7 \mathrm{mmol} / \mathrm{l}$; there was no ketonuria. Arterial blood gases, with the patient breathing air, were normal. No pathogens were cultured from his sputum and no acid fast bacilli seen. The Mantoux test response to 10 units purified protein derivative was negative.

Treatment was started with intravenous erythromycin and insulin and chest physiotherapy, with no improvement. Repeat chest radiographs showed worsening of the lower lobe shadowing. Fibreoptic bronchoscopy showed a white, cheese like mass occluding the lumen of the right lower lobe bronchus distal to the origin of the apical segment. Multiple 